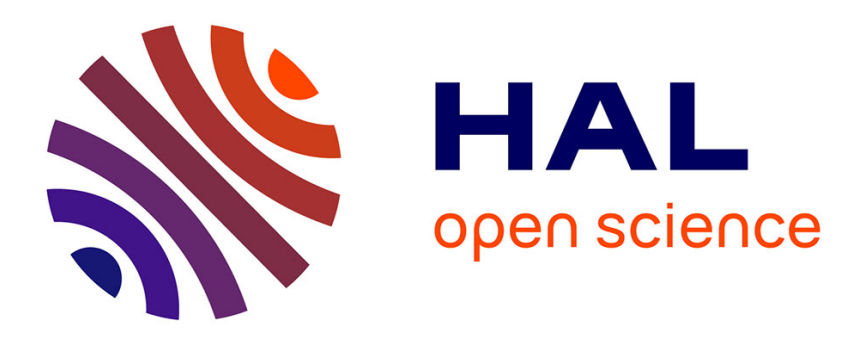

\title{
Status of the ANTARES Underwater Neutrino Telescope
}

\author{
G. D. Hallewell
}

\section{To cite this version:}

G. D. Hallewell. Status of the ANTARES Underwater Neutrino Telescope. Workshop On RICH Detectors: Dedicated to the Memory of Tom Ypsilantis (RICH 2002) 4, Jun 2002, Pylos, Greece. pp.138-144. in2p3-00012301

\section{HAL Id: in2p3-00012301 https://hal.in2p3.fr/in2p3-00012301}

Submitted on 16 Jun 2003

HAL is a multi-disciplinary open access archive for the deposit and dissemination of scientific research documents, whether they are published or not. The documents may come from teaching and research institutions in France or abroad, or from public or private research centers.
L'archive ouverte pluridisciplinaire HAL, est destinée au dépôt et à la diffusion de documents scientifiques de niveau recherche, publiés ou non, émanant des établissements d'enseignement et de recherche français ou étrangers, des laboratoires publics ou privés. 


\title{
Status of the ANTARES Underwater Neutrino Telescope
}

\author{
G. D. Hallewell \\ Centre de Physique des Particules de Marseille, \\ Representing the ANTARES Collaboration
}

\begin{abstract}
The ANTARES Collaboration is constructing a deep underwater neutrino detector for operation at $-2400 \mathrm{~m}$ off the French Mediterranean coast near Toulon. The detector, which will begin operation in 2004, will have an aperture of $\sim 0.1 \mathrm{~km}^{2}$, and will contain nine hundred photomultiplier tubes. The photomultiplier axes will be angled $45^{\circ}$ downward toward the seabed to observe the Čerenkov emissions of upward-going muons created by the interactions in or near the detector of high energy neutrinos traversing the Earth. These neutrinos arrive undeviated from a variety of galactic and extragalactic sources of astrophysical interest, and might be produced in the possible annihilation of dark matter neutralinos. The design and present status of the detector are summarized. Results from site evaluation and the development of supporting instrumentation are outlined.
\end{abstract}

\section{(1) Introduction}

The ANTARES $0.1 \mathrm{~km}^{2}$ underwater neutrino detector [1] is shown in artist's impression in figure (1). The first phase detector implementation in 2004 will have ten detection lines spaced on a $60 \mathrm{~m}$ sea floor grid, and will contain a total of nine hundred photo-multiplier tubes (PMTs) housed in optical modules ( $\$ 3)$, grouped in triplets at thirty levels rising from $-2300 \mathrm{~m}$ to -1950 m. A subsequent expansion of the array to fourteen such detector lines is possible.

Due to the purity of seawater at great depth, light absorption and scattering are small. However the detector is subject to backgrounds from cosmic ray muons, Čerenkov light from $\beta$ 's produced in ${ }^{40} \mathrm{~K}$ disintegrations, and luminescence from deep-sea creatures. Studies of these parameters have been made in several site evaluation campaigns $(\$ 2)$.

Signals from the PMT in each optical module will be processed by an "Analog Ring Sampler" (ARS) front end ASIC (\$4.2). Digitized pulse heights will be colour multiplexed (\$4.1) onto optical fibre up-links to be sent to the shore station via a $40 \mathrm{~km}$ multifibre electro-optic cable (laid October 2001) for on-shore filtering and event building.

Since each line will be suspended between a sea anchor and a submerged buoy subject to movement by deep ocean currents, the positions of individual OMs will be determined from measurements of line curvature and twist. Each line will be equipped with tilt-meters and compasses so that its movement can be logged. The positions of hydrophones mounted along the detection lines will be triangulated using a system of seabed acoustic transponders. A network of laser and LED beacons will be used to give redundancy in the on-line calibration of PMT timing.

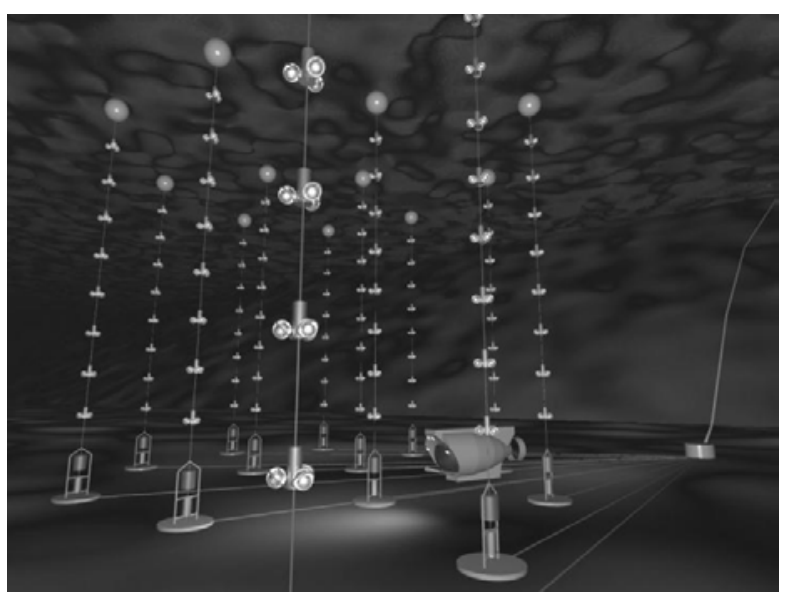

Figure (1) Artist's Impression of the ANTARES underwater neutrino detector array.

Many of the components of a large undersea Čerenkov detection array have been tested in a long immersion study (December 1999-June 2000) using a 350mdemonstration line equipped with 7 PMTs and a variety of instrumentation, including an acoustic triangulation system. The line was powered from and read out to the shore through an electro-optical cable with a single power conductor and return electrodes. More than 50,000 downgoing atmospheric muon tracks were successfully reconstructed from seven-fold coincidences, and algorithms for ${ }^{40} \mathrm{~K}$ background suppression were developed.

The following sections describe the principal components of the proposed detector in more detail.

\section{(2) ANTARES Site Evaluation}

An extensive series of site evaluation campaigns has been carried out by the ANTARES collaboration [2,3]. At the array depth of $2400 \mathrm{~m}$, the expected background rate from atmospheric cosmic ray muons is around 30 Hz. This rate is insignificant in comparison with the ${ }^{40} \mathrm{~K}$ background from sea salt, which adds a singles rate 
of $60 \mathrm{KHz}$ to each PMT, or with occasional bioluminescence [figure (2)], which can peak during short bursts up to $\mathrm{MHz}$ rates, and is seasonally- and ocean current dependent. In general, bioluminescence bursts are locally correlated between the PMTs in a particular triplet, while ${ }^{40} \mathrm{~K}$ background is not. The ARS ASIC has been designed to unravel single photoelectron signals from superimposed background (\$4.2).

Since the PMTs in the ANTARES array look downward, fouling is not a serious problem. The combined signal loss due to bio-deposition and sedimentation has been measured [2] to be less than $2 \%$ per year.

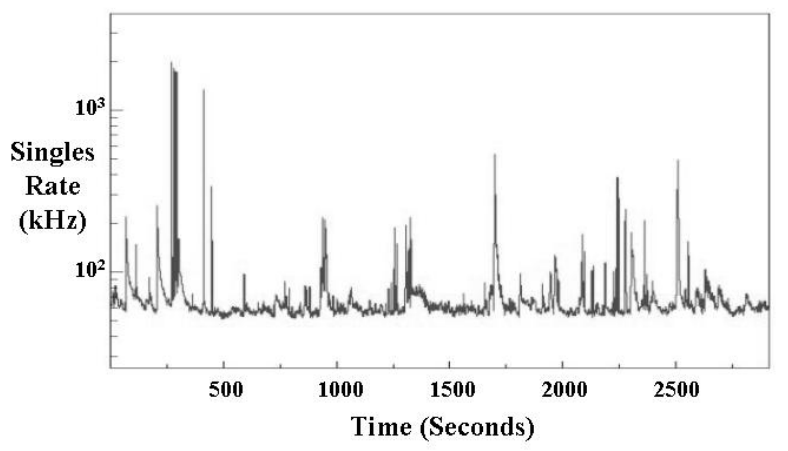

Figure (2) Background Singles Counting Rate in a $25 \mathrm{~cm}$ PMT at the ANTARES site.

The attenuation and scattering lengths in deep-sea water at the ANTARES site have been measured in several campaigns between 1998 and 2000. At 466nm, attenuation [scattering] lengths have varied between 48 and $61 \mathrm{~m}$ [231 and $305 \mathrm{~m}$ ]. The seasonal variation in these figures is significant, and the ANTARES array will incorporate an "instrumentation" line (§6.2), which will deploy instruments including transparency, sound velocity, salinity and current profile monitors.

\section{(3) The ANTARES Optical Module}

The construction of an optical module (OM) is illustrated in figure (3). All components are housed in a $43 \mathrm{~cm}$ diameter glass pressure sphere ${ }^{1}$ with a wall thickness of $1.5 \mathrm{~cm}$ and transmission $>87 \%$ for $\lambda>$ $400 \mathrm{~nm}$. The OM is based upon a Hamamatsu R708120 fourteen stage $25 \mathrm{~cm}$ PMT, whose principal parameters are shown in table (1). High voltage bias for the dynode array is generated on a custom PMT base from a $48 \mathrm{~V}$ input ${ }^{2}$, using a Cockcroft-Walton chain.

\footnotetext{
1 "VITROVEX" ® sphere by Nautilus Marine Service GmbH, D-28357 Bremen, Germany. Hemispheres of 3.3 low activity $(<0.5 \% \mathrm{~K}$ content $)$ borosilicate glass, $\mathrm{n}=1.47$.

${ }^{2}$ Mfr: ISEG Spezialelektronik GmbH, D-01454 Radeberg / OT Rossendorf, Germany
}

The PMT is optically coupled to and supported from the glass sphere by a silicone gel $^{3}$ [transmission $>88 \%$ for $(400<\lambda<700) \mathrm{nm}]$, and is shielded from the Earth's magnetic field by a two part mu-metal cage.

A light flasher system consisting of a blue LED and pulser circuit will monitor the PMT transit time. The LED is glued to the outside of the bulb and shines through it to illuminate the photocathode. The pulser is triggered from the detector master clock.

\begin{tabular}{|c|c|}
\hline Photocathode Sensitive area & $500 \mathrm{~cm}^{2}$ \\
\hline $\begin{array}{c}\text { Combined efficiency } \\
\text { (quantum } \oplus \text { collection) }\end{array}$ & $>16 \%$. \\
\hline Gain @ 2500 V. & $2 \times 10^{8}$ \\
\hline $\begin{array}{c}\text { Pulse amplitude } \\
\text { Nominal working gain of 5 x 107 }\end{array}$ & $\begin{array}{c}60 \mathrm{mV} \\
\text { into } 50 \Omega\end{array}$ \\
\hline Transit Time & $\sim 60 \mathrm{~ns}$ \\
\hline Transit Time Spread (TTS: FWHM) & $<3 \mathrm{~ns}$ \\
\hline $\begin{array}{c}\text { Dark count rate } \\
\text { ( 0.3 single photoelectron. threshold) }\end{array}$ & $<10 \mathrm{kHz}$. \\
\hline Pulse Rise time & $<5 \mathrm{~ns}$ \\
\hline Pulse Width (FWHM: single p.e.) & $<12 \mathrm{~ns}$ \\
\hline
\end{tabular}

Table (1) Parameters of the Hamamatsu 7081-20 PMT

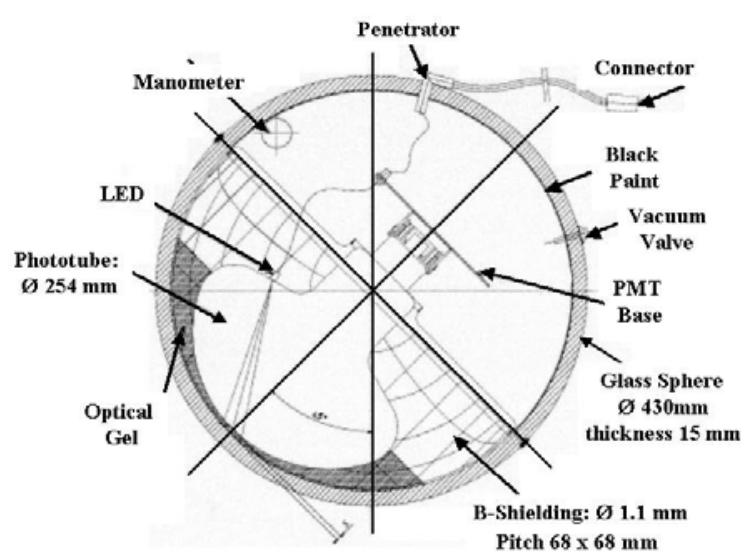

Figure (3) The Components of an Optical Module

\section{(4) The Detector Readout System}

\section{(4.1) General}

The functions of the detector readout system are to time stamp (accepted) analog PMT signals, to digitize their charge, and to merge the data from the ninety PMTs on each detector line onto each single fibre-optic uplink to the shore station. The first stage of PM signal processing is performed by the ARS ASIC (§4.2). The six ARS chips associated with each OM triplet (two per PMT), are housed in a common electronics container together with (depending on the position of

\footnotetext{
3 "Silgel” ® 612 A/B by Wacker. 2-component silicon rubber, room temperature cure: $\mathrm{n}=1.404$.
} 
the triplet along the detection line), the compass and tiltmeter and/or hydrophone signal processing cards of the line positioning system. Data from five PMT triplets are merged by a master DAQ card onto a single optical fibre descending to the seabed. At the base of each detection line the data from six descending fibres are combined onto a single fibre by dense wavelength division multiplexing (DWDM). These fibres pass through the junction box and onto the $40 \mathrm{~km}$ electrooptical cable linking the detector array to the shore.

\section{(4.2) The "Analog Ring Sampler"}

The ARS ASIC (figure (4)) is implemented in AMS $1.2 \mu \mathrm{m}$ CMOS technology, and is based on a fourchannel mixed analog / digital pipeline memory. The ARS also implements a waveform shape-sensitive discriminator to distinguish single photoelectron-like ("SPE") pulse shapes from superimposed pulses or from larger pulses characteristic of background.

The PMT direct anode signal enters one of the four inputs. Although each input can handle pulses up to $4.5 \mathrm{~V}$, an attenuated anode signal, and a signal from the $12^{\text {th }}$ dynode are used to extend dynamic range in the case of large signals. The distributed $20 \mathrm{MHz}$ master clock signal enters the fourth input.

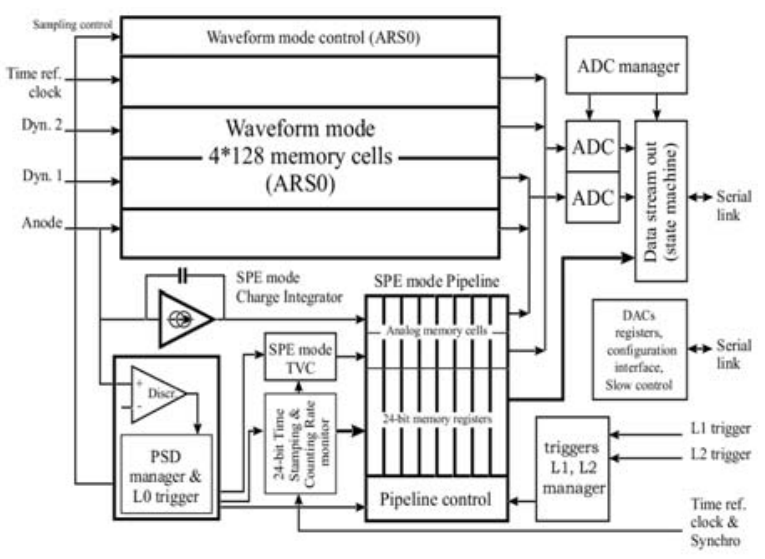

Figure (4) Functionality of the Analog Ring Sampler

A signal from the PMT anode triggers the ARS by crossing an amplitude threshold set to a fraction of the SPE average amplitude (the L0 threshold). The signal is time-stamped and its charge integrated to a precision of $\sim 10 \%$ to compensate for time walk effects. A time to voltage converter (TVC) interpolates between $20 \mathrm{MHz}$ clock pulses to give a time resolution of $\sim 0.4$ ns. Integrated charge and timestamp are then stored in the mixed analog/digital pipeline memory. At the ARS output, an SPE tag consists of a header (1 Byte), the integrated charge (1 Byte), TVC (1 byte) and the time stamp (3 Bytes).

In recent tests [6] with signals from a PMT illuminated with mainly single photoelectrons at a single point on the photocathode, a time resolution of $\sim 1.1 \mathrm{~ns}$ has been achieved, comparing favourably with the PMT TTS of $\sigma \sim 1.3 \mathrm{~ns}$ with full photocathode illumination. The intrinsic time resolution of the ARS was determined to be $\sigma \sim 350$ ps from measurement of the separation of two pulses injected into the ARS with a known time difference.

Analog pulse shape discrimination separates SPE pulses, which are charge integrated, from "waveform" (WF) pulse shapes, which are waveform-sampled in 128 samples at up to $1 \mathrm{GHz}$. This processing is implemented when the pulse height exceeds several photoelectrons, when the time over the L0 threshold is longer than normal, or when the L0 threshold is crossed more than once in the charge integration time. With 128 analog samples of the waveform, at the ARS output, a WF event can typically contain $>250$ bytes.

\section{(4.3) Data Bandwidth and Processing}

SPE events are expected to represent more than $98 \%$ of the ANTARES data, while WF events will be mainly generated by background phenomena, primarily ${ }^{40} \mathrm{~K}$ disintegrations and bioluminescence. The pipeline memory of each ARS can store up to 16 SPE hits or 4 WF hits.

With a singles rate of $70 \mathrm{kHz}$, and $2 \%$ fraction of WF events, the (typical) data rate will be $\sim 7 \mathrm{Mb} / \mathrm{s}$ per PMT. Two ARS chips are connected to each PMT to reduce dead time. This data rate is shared between the (fast) output ports of the two ARSs and is well within the $20 \mathrm{Mb} / \mathrm{s}$ bandwidth limit of each. The PMT readout system is intended to handle an average singles rate of up to $100 \mathrm{kHz}$ with surges of up to $250 \mathrm{kHz}$ due to bioluminescence bursts.

Data from five PMT triplets (five levels) will be merged onto a single descendant fibre with a typical bandwidth of $105 \mathrm{MB} / \mathrm{s}$. Data from six such fibres will be DWD-multiplexed onto a single fibre at the bottom of each detection line, resulting in a typical data rate to shore of $700 \mathrm{MB} / \mathrm{s}$ per line.

An on-shore data switchyard will de-multiplex up to 70 incoming data streams and pass data to a processing farm consisting of up to $100 \mathrm{PCs}$ running at an input data rate in the range $50-100 \mathrm{MB} / \mathrm{s}$.

\section{(5) The Detector Positioning System}

\section{(5.1) Specifications}

The reconstruction of muon tracks in the ANTARES detector is based on precise measurements $(\sim 1 \mathrm{~ns})$ of the arrival times of Cerenkov photons at OMs. This reconstruction requires knowledge of the positions of the OMs relative to each other, or more practically, 
with respect to fixed reference points such as the detector line anchors. The precision of this spatial positioning should be better than the dispersive uncertainty in the arrival time of Čerenkov light detection $(\sim 1.6 \mathrm{~ns}$ over a typical flight distance of $40 \mathrm{~m})$. Since $1 \mathrm{~ns}$ is equivalent to $22 \mathrm{~cm}$ of light travel path in water, we aim to measure the relative position of every $\mathrm{OM}$ in the detector to $\sim 10-20 \mathrm{~cm}$.

The relative positions of the OMs will be obtained from fits to position data determined by two independent systems: a high frequency long baseline acoustic system [7] and a series of semiconductor tiltmeter-compass sensors disposed along each detection line. The relative positions of the OMs will then be deduced from this reconstructed line shape and from the geometry of the OM frame.

\section{(5.2) The Acoustic Positioning System}

An array of $40-60 \mathrm{kHz}$ acoustic transponders $[7]^{4}$ will be deployed on the seabed (four delineating a $300 \mathrm{x}$ $300 \mathrm{~m}$ square around the site, and one on the sea anchor of each detection line), and will transmit sound signals to hydrophones mounted at six positions (altitudes 100, $184,256,328,388 \& 448 \mathrm{~m}$ ) along each detection line. The $3 \mathrm{D}$ positions of the hydrophones will be obtained by triangulation. The sea bed transponders are able to interrogate each other to determine their own relative positions.

The transmission and reception of each sound pulse will be time-stamped relative to the $20 \mathrm{MHz}$ distributed master clock. The conversion of acoustic transit times to distances requires accurate knowledge of the sound velocity within the detector. This in turn depends on the temperature, salinity and pressure [8]. Instrumentation to monitor these parameters will be included on a dedicated instrumentation line $(\S 6.2)$.

\section{(5.3) The Tiltmeter/Compass system}

The detection lines will incorporate combined bi-axial tiltmeter and compass sensors ${ }^{5}$ to give the local tilt angles of each OM triplet level with respect to the horizontal plane (range [precision]; $\pm 20^{\circ}\left[ \pm 0.2^{\circ}\right]$ in pitch and roll), as well as its orientation relative to Earth Magnetic North (heading range [precision]; 0$\left.360^{\circ}\left[ \pm 0.5 \rightarrow 1^{\circ}\right]\right)$.

We have simulated the performance of the positioning system in various configurations (varying the number of acoustic sensors and tiltmeters; the locations and precision of sensors, positions of missing sensors etc.), with differing values of sea water current

\footnotetext{
${ }^{4}$ Mfr: Genisea/ECA, 83078 Toulon, France: Frequency range 44$60 \mathrm{kHz}, 2 \mathrm{kHz}$ channel spacing

${ }^{5}$ Model TCM2-20: Mfr: Precision Navigation Inc., Santa Rosa CA95403, USA http://www.pnicorp.com
}

and detector line twist as a function of altitude. Our studies indicate that the proposed combination of acoustic triangulation and tiltmeter-compasses should allow the positions of all OMs to be determined with an accuracy $\sigma<10 \mathrm{~cm}$ in sea water currents up to 15 $\mathrm{cms}^{-1}$.

\section{(6) Detector Infrastructure}

The ANTARES detector array, located in deep water offshore, will be supplied with electrical power, controlled and monitored from land, and will transmit physics and calibration data to onshore facilities for storage and analysis through a $40 \mathrm{~km}$ electro-optical telecommunications cable ${ }^{6}$. The cable [table (2)] contains 48 fiberoptic links and a single electrical conductor through which the detector will be powered with $4500 \mathrm{~V} 50 \mathrm{~Hz} \mathrm{AC}$, the current return to the shore being through sea and shore electrodes.

\begin{tabular}{|c|c|}
\hline Communication Function & $\begin{array}{c}\text { Wires/ } \\
\text { Fibres }\end{array}$ \\
\hline $\begin{array}{c}\text { Detector Power Electrical Conductor } \\
\text { (4400V AC 50 Hz: 8 Amperes) }\end{array}$ & 1 wire \\
\hline $\begin{array}{c}\text { DAQ Tx (0-15) (data uplinks from up to 16 } \\
\text { detector/ instrumentation lines) }\end{array}$ & 16 \\
\hline $\begin{array}{c}\text { DAQ Rx (0-15) (data/configuration downlinks to } \\
\text { up to 16 detector/instrumentation lines) }\end{array}$ & 16 \\
\hline JB Slow Control System Rx (3 redundant systems) & 3 \\
\hline JB Slow Control System Tx (3 redundant systems) & 3 \\
\hline JB Slow Control System Power Supply Enables & 2 \\
\hline Clock Distribution (4 redundant systems) & 4 \\
\hline Spare Fibres & 4 \\
\hline
\end{tabular}

Table (2) Main electro-optical cable functionality

Each detection line is held taut between a buoy of syntactic foam ${ }^{7}$ (glass micro-spheres in resin: $6370 \mathrm{~N}$ buoyancy at $-2500 \mathrm{~m}$ ) and a concrete seabed anchor. The line may be detached from its sea anchor via an acoustic release system. A coded signal received at a hydrophone mounted on the anchor triggers a release shackle ${ }^{8}$ and allows the line to float to the surface for recovery.

The hub of the detector array is a central titanium junction box (JB), which splits the power between the detector lines, distributes clock signals and gathers the data signals from the detector lines onto the main electro-optical cable for transmission to the shore. The JB contains a $24 \mathrm{~kW}$ transformer, across whose primary are $4500 \mathrm{~V}$ input from the cable and the sea potential via an iridium return electrode. Sixteen separate $500 \mathrm{~V}$

\footnotetext{
${ }^{6}$ Manufactured by Alcatel; laid by Alcatel October 2001

${ }^{7}$ Mfr: BMTI s.a. 83500 La Seyne sur Mer, France

8 Model RT861-B2T: Mfr: Oceano Technologies, 29200 Brest, France http://www.oceano-technologies.fr
} 
secondaries power and galvanically isolate each detection or instrumentation line. Each output can be switched on/off or reset by a remotely controllable circuit breaker handled via the triply-redundant JB slow control system. Passive fibreoptic splitters distribute the master clock signals to the sixteen output lines.

Outputs from the junction box, each containing two electrical conductors and four optical fibres (2 CLOCK, DAQ-Tx, DAQ-Rx) terminate in wetmateable connectors ${ }^{9}$, as do the power and fibre-optic links at the bottom of each detection line. Lines will be connected to the JB as they are deployed, using a remotely-controlled submarine vehicle (ROV) equipped with a manipulator arm.

Power arriving at a detection line from the JB is converted from $500 \mathrm{~V}$ AC to $380 \mathrm{~V}$ DC for passage up the cable to the thirty electronics containers. In these, it is further reduced via DC/DC converters for use in optical modules and the readout electronics.

\section{(6.2) Instrumentation line}

One of the sixteen JB outputs will be reserved for an "instrumentation line" containing equipment for monitoring the detector array and deep-sea environmental parameters including salinity, sound velocity, water transparency and current profile. For detector-wide timing calibration, a laser beacon will illuminate the array with fast (900ps), time-stamped laser ${ }^{10}$ pulses at a wavelength of $456 \mathrm{~nm}$. Its action will complement shorter-range blue LED beacons distributed among the detection lines.

The deep-sea current profile (velocity, direction) will be sampled over 256 intervals over the full detector depth of $300 \mathrm{~m}$ by a pair of $300 \mathrm{kHz}$ acoustic Doppler current profilers $^{11}$ with a velocity range [resolution] of $5 \mathrm{~ms}^{-1}\left[1 \mathrm{mms}^{-1}\right]$.

The optical attenuation of water at $470 \mathrm{~nm}$ will be measured over a $25 \mathrm{~cm}$ path length using a commercial photodiode-based deep-sea transmission meter ${ }^{12}$. The line will be equipped with sound velocity meters of the same type as installed close to the sea anchors of some detection lines. These devices ${ }^{13}$ have a flight path of 20 $\mathrm{cm}$ and a precision of $\pm 0.05 \mathrm{~ms}^{-1}$ for typical velocities in the range $1400-1600 \mathrm{~ms}^{-1}$. Several velocity meters

\footnotetext{
${ }^{9}$ Mk II Hybrid Connector: Mfr: Ocean Design Inc, Ormond Beach, FL32174, USA

${ }^{10}$ Incorporating a NG-10120-120 Green (532 nm) laser head. Mfr

Nanolase, 38941 Meylin, France

${ }^{11}$ Workhorse Monitor; Mfr: RD Instruments, San Diego CA 92131 USA

${ }^{12}$ C-STAR by WETlabs Inc. http://www.wetlabs.com

${ }^{13}$ Mfr: Genisea, France: model QUUX-3A(A):
}

will also be equipped with conductivity-temperature ${ }^{14}$ and depth (pressure) probes ${ }^{15}$.

\section{(7) Conclusion and Schedule}

The ANTARES project has finished its underwater site evaluation and instrumentation development, and has entered the construction and deployment phase. In October 2001 the $40 \mathrm{~km}$ underwater cable was successfully laid. The construction of the 900 optical modules for the deployment of ten detection lines is well advanced. The analog ring sampler front end ASIC has met specification. The commissioning of a full readout chain is well advanced, and a preproduction "sector line" of five OM triplets will be deployed in late 2002 together with the electro-optical junction box and an instrumentation line. The deployment of full detector lines is expected to commence in 2003, and the commissioning of a $0.1 \mathrm{~km}^{2}$ array of ten detection lines is planned for completion late in 2004.

\section{References}

[1] ANTARES Technical Design Report, version 1.0, July 2nd 2001 : via http://antares.in2p3.fr

[2] P. Amram et al; Sedimentation and Fouling of Optical Surfaces at the ANTARES Site, June 2002, submitted to Astroparticle Physics astro-ph/0206454

[3] P.Amram et al ; Background light in potential sites for the ANTARES undersea neutrino telescope Astroparticle Physics 13 (2000) 127-136

[4] P. Amram et al; The ANTARES Optical Module; NIM A 484 (2002) 369

[5] F. Druillole et al; Proc. IEEE Nuclear Science Symposium, 4-10 Nov 2001, San Diego, CA, USA

[6] F. Feinstein; "The Analogue Ring Sampler: a frontend chip for ANTARES."

Proc. 2002 Conference on advances in Photon Detection, Baune, France, July 2002

[7] V. Bertin, Genisea ; "Système de Positionnement Acoustique base longue: Description Technique" ANTARES Report 3INS0103B, May 2000

[8] C. Chen and F Millero; "Speed of sound in sea water at high pressures," J. Acoust. Soc. Am., Vol. 62, No. 5, 1129-1135, Nov 1977

\footnotetext{
${ }^{14}$ OEM-OT sensor by Falmouth Scientific Inc., Cataumet MA02534: http://www.falmouth.com precision $\pm 0.001 \mathrm{mScm}^{-1}$

${ }^{15}$ Mfr: Druck S.A., 92600 Asnières sur Seine, France
} 